\section{Clinical features of thyroid-associated ophthalmopathy in clinically euthyroid Korean patients}

\begin{abstract}
Aim To compare clinical characteristics and thyroid-stimulating hormone receptor antibodies (TRAbs) in thyroid-associated ophthalmopathy (TAO) in euthyroid Korean patients with those in hyperthyroid patients. Methods Clinical activity scores (CASs), modified NOSPECS scores, exophthalmometry values, prevalence of optic neuropathy, restrictive myopathy and lid retraction, and the positivity and levels of TRAb (thyrotropin-binding inhibitor immunoglobulin (TBII) and thyroidstimulating immunoglobulin (TSI)) were compared in 24 euthyroid (group A) and 139 clinical/subclinical hyperthyroid TAO patients (group B).
\end{abstract}

Results Group A presented more clinically unilateral involvement than group B $\mathbf{( 7 9 . 2 \%}$ vs $27.3 \%, P<0.001$ ), less active (CAS 1.50 vs 2.26, $P=0.014)$ and less severe clinical course (NOSPECS 3.38 vs $4.13, P=0.037$ ). Lid retraction was more prevalent in group $\mathrm{A}$ than group B $(91.7 \%$ vs $66.2 \%, P=0.014)$. Prevalence of optic neuropathy and restrictive myopathy, and the mean value of exophthalmometry were not different. Mean TBII levels were lower $(7.20 \mathrm{IU} / \mathrm{l})$ in group $\mathrm{A}$ than in group $B(44.58 \mathrm{IU} / \mathrm{l}, \mathrm{P}<\mathbf{0 . 0 0 1 )}$. A similar difference was found in the TSI bioassay (201.40\% vs $425.19 \%, P=0.001)$. The positive rate of TBII in group A $(34.8 \%)$ was

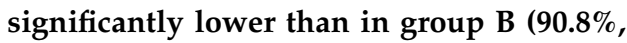
$P<0.001)$. The positive rate of TSI was high in both group A (83.3\%) and B (91.7\%), with no significant difference $(P=0.337)$.

Conclusions Patients with euthyroid TAO showed a less active and severe clinical course, more unilateral involvement, and lower levels of TRAb than those in patients with hyperthyroid TAO. These distinct
SY Jang ${ }^{1}$, SY Lee ${ }^{1}$, EJ Lee ${ }^{2,3}$ and JS Yoon ${ }^{1}$

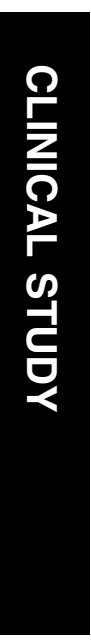

clinical and biochemical characteristics might be useful in assessment of euthyroid TAO, and the TSI might be more sensitive for diagnosing these patients.

Eye (2012) 26, 1263-1269; doi:10.1038/eye.2012.132; published online 29 June 2012

Keywords: thyroid-associated ophthalmopathy; euthyroid; hyperthyroid; TSH-receptor antibody

Introduction

A few case reports of thyroid-associated ophthalmopathy (TAO) without hyperthyroidism were reported several decades ago. ${ }^{1,2}$ These patients presented with the ocular signs of TAO and had normal thyroid function without any clinical symptoms of hyperthyroidism. They were described as having euthyroid Graves' orbitopathy (GO) or euthyroid TAO. However, definite diagnostic criteria for euthyroid TAO have yet to be established. ${ }^{3,4}$

Diagnosis of euthyroid TAO is often difficult, because of the absence of the standard criteria, absence of confirmatory diagnostic tests, and rarity of cases. As TAO occurs in a close temporal relationship with hyperthyroidism, ${ }^{5}$ it is reasonable that TAO is rare in patients with normal or hypothyroid function. Khoo et al, ${ }^{6}$ in studies conducted in Singapore using 1020 Asian patients with GO, reported that the prevalence of euthyroid TAO was $0.7 \%$ and hypothyroid TAO was $0.2 \%$. Thus, euthyroid TAO is rarely encountered in a clinical situation, making it difficult to diagnose.

A few studies have reported that a patient could be diagnosed with TAO, even with euthyroid status. ${ }^{7-9}$ Because most cases
${ }^{1}$ Department of Ophthalmology, Institute of Vision Research, Yonsei University College of Medicine, Seoul, Korea

${ }^{2}$ Department of Endocrinology, Brain Korea 21 Project for Medical Science, Institute of Endocrine Research, and Severance Integrative Research Institute for Cerebral and Cardiovascular

Disease, Seoul, Korea

${ }^{3}$ Department of Biochemistry and Molecular Biology, Yonsei University College of Medicine,

Seoul, Korea

Correspondence:

JS Yoon, Department of Ophthalmology, Institute of Vision Research, Yonsei University College of Medicine, 50 Yonsei-Ro, Seoul 120-752, Korea Tel: + 8222228 3570; Fax: + 8223120541 E-mail: yoonjs@yuhs.ac

Received: 17 January 2012 Accepted in revised form: 2 May 2012 Published online: 29 June 2012 Seodaemun-gu, 
involving euthyroid TAO showed no thyroid abnormalities except testing positive for thyroidstimulating hormone receptor antibodies (TRAbs), 7,9 the clinical significance of TRAb as a criterion in the diagnosis of euthyroid TAO has been suggested. ${ }^{3,6}$

Recently, Eckstein et al ${ }^{10}$ reported that the TRAb titer was low in euthryoid and hypothyroid patients, suggesting that a more sensitive assay technique was necessary to utilize the TRAb assay for diagnosis of euthyroid/hypothyroid TAO. The TRAb assay used in this report was the thyrotropin-binding inhibitor immunoglobulin (TBII) assay, which cannot discriminate between thyroid-blocking immunoglobulin and thyroidstimulating immunoglobulin (TSI). Using a secondgeneration TBII assay, Eckstein et al ${ }^{10}$ reported that the positive rate of TRAb in the euthyroid/hypothyroid TAO patients was $69 \%$.

In another study by Kazuo et $a l^{3}$ using Asian patients, the TSI measurement was more sensitive than the TBII assay used in the diagnosis of euthyroid TAO. The TSI assay measures the production of cyclic adenosine monophosphate in response to a thyrotropin (TSH)receptor interaction with TSI. The prevalence of TRAb in euthyroid TAO patients was $28.6 \%$ using the firstgeneration TBII assay and $82.9 \%$ with the TSI assay using cultured rat thyroid cells.

In the present study, we investigated the clinical and biochemical characteristics of Korean euthyroid TAO patients by comparing them with hyperthyroid TAO patients. In the same study, we also assessed the ability of the TBII and TSI assays to diagnose TAO.

\section{Materials and methods}

\section{Study design}

This was a retrospective observational study. The participants in this study were patients with TAO who visited the Department of Ophthalmology, Severance Hospital of Yonsei University College of Medicine, between January 2008 and December 2010. The research conducted in this study adhered to the tenets of the Declaration of Helsinki and was approved by the Institutional Review Board at the Yonsei University College of Medicine.

\section{Patients}

A single observer (JSY) diagnosed and conducted the follow-up of all patients. Diagnosis of TAO was based on clinical ophthalmic examination. All patients had clinical features of the disease including at least one of the following in one eye (unilateral involvement) or both eyes (bilateral involvement): lid retraction (upper eyelid margin at or above the superior corneoscleral limbus in primary gaze or $2 \mathrm{~mm}$ of difference on lid aperture in primary gaze), ${ }^{3,11}$ soft tissue involvement, exophthalmos (Hertel measurement $\geq 17 \mathrm{~mm}$ and/or $2 \mathrm{~mm}$ of difference between the eyes), ${ }^{3}$ restrictive myopathy (limitation of eye movement in the Hess chart and/or diplopia assessed by binocular single-vision test and/or objective evidence of enlarged muscles assessed by computed tomography (CT)), and optic neuropathy (abnormal visual acuity, relative afferent pupillary defect, decreased color perception, and visual field by perimetry).

Hertel measurement $\geq 17 \mathrm{~mm}$ was chosen as the cut off value of exophthalmos based on several reports in which the normal value of exophthalmometry for Asians was lower than that of Caucasians. 3,12,13

Thyroid carcinoma, Hashimoto thyroiditis (clinical/ subclinical hypothyroidism), and any other possible causes for ocular manifestations were carefully ruled out based upon patient history, examinations including CT or thyroid scans, and laboratory tests. All the patients were imaged by CT scans to evaluate carefully and objectively to define unilaterality.

The medical records of the remaining 163 patients were retrospectively reviewed. Patient history, including hyperthyroidism, family history, smoking history, duration after first symptom of TAO, duration of thyroid disease, and laboratory peripheral thyroid function, was examined.

\section{Grouping of the patients}

The diagnosis of euthyroid patients was based upon the presence of normal serum fT4, T3, and TSH concentration without a clinical history of hyperthyroidism (group A).

The diagnosis of hyperthyroid TAO (group B) was established using followings criteria:

(1) Elevated serum T3 and free T4 concentration (clinical hyperthyroid TAO),

(2) Normal serum T3, free T4 levels but decreased TSH concentration (subclinical hyperthyroid TAO),

(3) A history of antithyroid treatment if being treated with an antithyroid drug or having had previous antithyroid treatment including surgery or radioiodine. The patient was also assigned to the hyperthyroid TAO group.

Remission of Graves' disease (GD) was defined as at least 1 year of euthyroidism after cessation of antithyroid treatment. We included the patient who presented with remission of hyperthyroidism and typical TAO signs in the group B. Patients with elevated TSH (clinical and 
subclinical hypothyroidism) were excluded from the present study.

\section{Methods}

Eye examinations were performed by an ophthalmologist (JSY) for the following criteria, using a modified European Group on Graves' Orbitopathy case record form: eyelid aperture size (measured in $\mathrm{mm}$ ), any soft tissue involvement, exophthalmos (measured in mm using a Hertel exophthalmometer (Oculus; Oculus Optik Geraete, Wetzlar, Germany), extraocular muscle (EOM) involvement (measured in degrees of restriction with the Hess chart and measured in the degree of diplopia by the binocular single-vision test), and visual acuity. To reduce inter and intra observer variability, all subjects were examined by the same clinician at every visit and were repeatedly assessed using the single exophthalmometry. ${ }^{14}$ We used the median value of exophthalmometry among repeated measurements.

Clinical activity of the disease was assessed using a clinical activity score (CAS) based upon seven signs of orbit inflammation. ${ }^{15}$ The assessment items were: spontaneous retrobulbar pain, pain on eye movement, redness of the eyelid, conjunctival injection, chemosis, swelling of the caruncle, and swelling of the eyelids. If the patient had any of these symptoms, one point was assigned for each item, with a total possible score of 0-7 points. The severity of GO was described by the modified NOSPECS score obtained by adding NOSPECS grades for each of the followings: lid retraction, soft tissue involvement, exophthalmos, EOM involvement, corneal defect, and optic nerve compression. We used the modified NOSPECS classification, which was used as reported by Eckstein et al. ${ }^{16}$

\section{Outcome variables}

The following parameters were compared between the two groups: age, sex ratio, smoker proportion, duration of ocular symptoms, bilateral or unilateral involvement, CAS, modified NOSPECS score, prevalence of optic neuropathy, prevalence of restrictive myopathy, the mean value of exophthalmometry and prevalence of lid retraction, and mean TBII and TSI bioassay levels. The TBII and TSI bioassays were performed at the initial visit.

\section{TRAb measurements}

TRAb was measured at the first visit and at regular intervals during follow-up, using the first-generation TBII assay. The Thyrotropin Receptor Autoantibodies Kit (RSR Ltd, Cardiff, UK) was used according to the manufacturer's instructions. This kit consists of the TSH receptor and ${ }^{125} \mathrm{I}$-labeled TSH. Quantification of serum TBII was based upon TRAb inhibition of radiolabeled TSH binding to porcine thyroid membrane extracts. Quantitation of TSI was based upon the Mc4-CHO TSI assay. TSI in patient sera was measured using the ThyretainTM TSI Reporter BioAssay (Diagnostic Hybrids, Inc., Athens, OH, USA) according to the manufacturer's instructions.

\section{Data analysis and statistics}

The normality of data distribution for all variables was tested using the Kolmogorov-Smirnov test. We used independent $t$-tests and the Chi-square test to compare primary data and ascertain normality of age and laboratory distribution between the two patient groups. The Wilcoxon rank-sum test was used to compare values, which did not conform to a normal distribution, such as onset of ocular symptom, CAS, NOSPECS, and the value of exophthalmometry.

\section{Results}

Of a total of 163 patients, 139 patients were diagnosed with hyperthyroidism and 24 patients with euthyroid.

\section{Comparison between the two groups}

In a demographic comparison of the two groups, there were no statistical differences in age, smoker proportion, and duration of ocular symptoms (Table 1). There was a greater percentage of men in group $A$ than in group $B$ (41.7\% vs $20.9 \%, P=0.038$ ). The onset time of ocular symptoms was a median of 3 months in both groups, revealing no statistical difference $(P=0.918)$.

Clinically unilateral eye involvement developed in $79.2 \%$ in group A and $27.3 \%$ in group B, revealing a statistically significant difference $(P<0.001)$ (Table 2$)$. In comparing the CAS and NOSPECS scores, group A was less active (CAS 1.50 vs 2.26, $P=0.014$ ) and was associated with a less severe clinical course (NOSPECS 3.38 vs $4.13, P=0.037$ ) than group B (Table 2). Lid retraction, the most common ocular sign of TAO, was observed in $91.7 \%$ of group A, showing a significantly higher rate than that in group $\mathrm{B}(66.2 \%, P=0.014)$. There was no difference in compressive optic neuropathy, EOM involvement, and exophthalmos between the two groups.

Among the 24 patients with euthyroid TAO, lid retraction was not observed in only 2 patients. One patient showed $20 \mathrm{~mm}$ exophthalmos of eyes as measured by Hertel exophthalmometry and the other patient showed $3.5 \mathrm{~mm}$ exophthalmos and restrictive myopathy limited to the right eye. 
Table 1 Comparison of patient demographics between euthyroid (group A) and hyperthyroid TAO (group B)

\begin{tabular}{lccc}
\hline & Group A & Group B & P-value \\
\hline Number of patients & 24 & 139 & \\
Age (mean \pm SD) & $44.4 \pm 11.6$ & $42.2 \pm 13.5$ & 0.444 \\
Sex (number of male (\%)) & $10(41.7)$ & $29(20.9)$ & $0.038^{\mathrm{a}}$ \\
Smoker (number (\%)) & $8(33.3)$ & $32(23.0)$ & 0.278 \\
Duration of eye symptom (median (IQR)) & 3.0 months (4.75) & 3.0 months (9.5) & 0.918 \\
Duration of Graves' disease (median (IQR)) & N/A & 10 months (21.0) \\
\hline
\end{tabular}

Abbreviation: TAO, thyroid-associated ophthalmopathy.

Independent $t$-test for comparisons of age.

Wilcoxon signed rank-sum test for comparisons of duration of ocular symptoms.

Chi-square test for comparison of sex ratio and smoker proportion.

${ }^{a} P<0.05$ was considered statistically significant.

Table 2 Comparison of ocular manifestations between euthyroid (group A) and hyperthyroid TAO (group B)

\begin{tabular}{|c|c|c|c|}
\hline & Group $A(\mathrm{n}=24)$ & Group B $(\mathrm{n}=139)$ & P-value \\
\hline & & Remission of hyperthyroidism $(\mathrm{n}=10)$ & \\
\hline \multirow[t]{2}{*}{ Asymmetric involvement (number (\%)) } & $19(79.2 \%)$ & $38(27.3 \%)$ & $<0.001^{\mathrm{a}}$ \\
\hline & & $3(30.0 \%)$ & $0.015^{\mathrm{a}}$ \\
\hline \multirow[t]{2}{*}{ Clinical activity score (mean (SD)) } & $1.50(1.3)$ & $2.26(1.7)$ & $0.014^{\mathrm{a}}$ \\
\hline & & $1.10(1.7)$ & 0.381 \\
\hline \multirow[t]{2}{*}{ NOSPECS score (mean (SD)) } & $3.38(1.4)$ & $4.13(2.4)$ & $0.037^{\mathrm{a}}$ \\
\hline & & $2.60(1.3)$ & 0.196 \\
\hline \multirow[t]{2}{*}{ Optic neuropathy (number (\%)) } & $0(0 \%)$ & $8(5.7 \%)$ & 0.216 \\
\hline & & $0(0 \%)$ & - \\
\hline \multirow[t]{2}{*}{ Restrictive myopathy (number (\%)) } & $6(25.0 \%)$ & $43(30.9 \%)$ & 0.558 \\
\hline & & $3(30.0 \%)$ & 0.667 \\
\hline \multirow[t]{2}{*}{ Exophthalmometry value (mean (SD)) } & $17.04(3.71)$ & $18.56(3.19)$ & 0.149 \\
\hline & & $16.40(2.56)$ & 0.322 \\
\hline \multirow[t]{2}{*}{ Lid retraction (number $(\%)$ ) } & $22(91.7 \%)$ & $92(66.2 \%)$ & $0.014^{\mathrm{a}}$ \\
\hline & & $5(50.0 \%)$ & $0.014^{\mathrm{a}}$ \\
\hline
\end{tabular}

Abbreviation: TAO, thyroid-associated ophthalmopathy.

Wilcoxon rank-sum test for comparison of clinical activity score, NOSPECS score, and the mean value of exophthalmometry.

Chi-square test or Fisher's exact test for comparison of categorical variables.

${ }^{a} P<0.05$ was considered statistically significant.

We compared the clinical parameters of euthyroid TAO (group A) with a subgroup of 10 patients included in group B, who were classified as reaching remission after cessation of antithyroid drug therapy for GD. There was no statistical difference in age, sex ratio, proportion of smokers, and duration of ocular symptoms (data not shown). Mean CAS and modified NOSPECS scores were lower in the subgroup of group B than in group A, however, the difference was not significant (Table 2). Similar to the comparative results between group A and total group B, clinically asymmetrical involvement and lid retraction were significantly more prevalent in group A than in the subgroup of group B.

Mean TBII levels were significantly lower (7.20 IU/1) in group A than in group B $(44.58 \mathrm{IU} / 1, P<0.001)$, as was the TSI assay (201.4 vs 425.19 specimen-to-reference ratio $(\mathrm{SRR} \%), P=0.002)$ (Table 3$)$. The prevalence of TBIIpositive patients in group A $(34.8 \%, 8 / 23)$ was significantly lower than that in group B $(90.8 \%, 113 / 119$, $P<0.001)$. The results of the TSI assay were high in both group A $(83.3 \%, 15 / 18)$ and group B $(91.7 \%, 55 / 60)$, with no statistically significant difference $(P=0.337)$

(Figure 1).

Among the 24 patients with euthyroid TAO, there were 4 patients who complained that the ocular symptoms started more than 12 months previously. Both TRAb assays were negative in three patients, while a 59-yearold man who presented unilateral lid retraction showed a 9.62 (borderline) IL/1 for TBII and 176.2 (positive) SRR\% for TSI. The three patients for whom both TBII/TSI assays were negative had typical TAO ocular signs. Two of the patients had only unilateral lid retraction and the other had EOM involvement causing $3.5 \mathrm{~mm}$ proptosis. We followed up each patient for 19, 9, and 12 months, respectively. Two patients presenting with long-standing unilateral eyelid retraction had no previous history of 
Table 3 Comparison of serological data between euthyroid (group A) and hyperthyroid TAO (group B)

\begin{tabular}{lccr}
\hline & Group A & Group B & P-value \\
\hline T3 (ng/dl) mean \pm SD & $1.65 \pm 1.60$ & $2.58 \pm 1.74$ & $0.046^{\mathrm{a}}$ \\
Free T4 (ng/ml) & $1.20 \pm 0.22$ & $3.01 \pm 3.19$ & $<0.001^{\mathrm{a}}$ \\
TSH (mU/l) & $1.62 \pm 1.21$ & $0.90 \pm 3.41$ & 0.350 \\
TBII (IL/l) & $7.20 \pm 10.90$ & $44.58 \pm 27.75$ & $<0.001^{\mathrm{a}}$ \\
TSI (SRR\%) & $201.4 \pm 175.85$ & $425.19 \pm 204.95$ & $0.001^{\mathrm{a}}$ \\
\hline
\end{tabular}

Abbreviations: SRR, specimen-to-reference ration; TBII, thyrotropinbinding inhibitor immunoglobulin; TSI, thyroid-stimulating immunoglobulin.

${ }^{\mathrm{a}} P<0.05$ was considered statistically significant. The independent $t$-test was used.

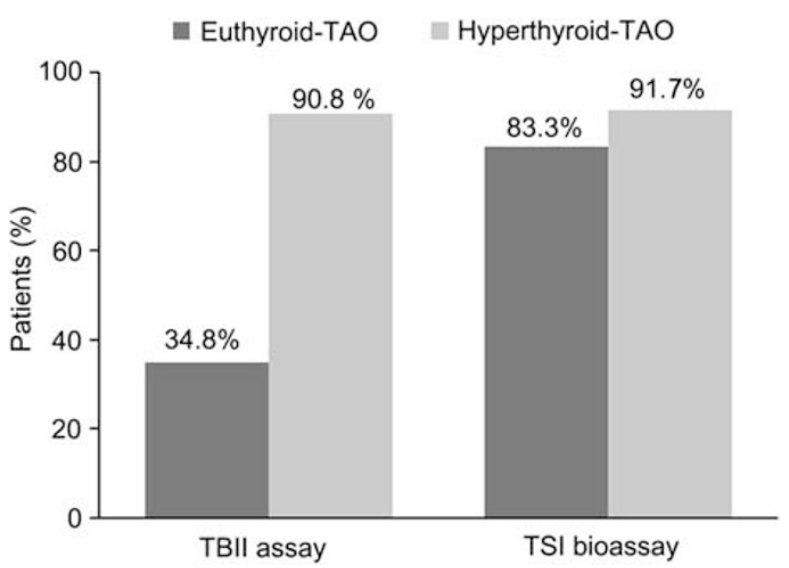

Figure 1 Thyrotropin-TBII and TSI assays. The percentage of positive results using the TBII assay in group A $(34.8 \%, 8 / 23)$ was significantly lower than that in group B $(90.8 \%, 113 / 119$, $P<0.001$ ); however, the percentage of positive results of the TSI assay was high in both group A $(83.3 \%, 15 / 18)$ and B $(91.7 \%, 55 /$ $60)$, with no statistically significant difference $(P=0.337)$.

systemic disease and other evidence of pseudoretraction. ${ }^{17}$ There were no changes in ocular signs during the follow-up period. The other patient, a 45-year-old male presenting with proptosis and diplopia, had right inferior and medical rectus muscle enlargement on CT scan. Imaging revealed well-defined fusiform enlargement posteriorly, with no anterior tendon involvement.

Among the remaining 20 patients with ocular symptoms less than $<12$ months, either the TBII or TSI assay was positive, with TBII positive in $42.1 \%$ (8/19) of the patients and TSI positive in 100\% (14/14) of the patients.

\section{Clinical course of the euthyroid group}

A total of 24 patients with euthyroid TAO were followed up for a mean period of 9.3 months (median 7.0 months, range 3-42 months). During follow-up, subclinical hypothyroidism developed in three patients, whereas the other 21 patients showed no changes in the thyroid function test (TFT).

\section{Discussion}

TAO, an inflammatory condition affecting the orbit, frequently accompanies GD. Pathogenic ocular involvement in GD can be explained by TSHR expression not only in the thyroid follicular cells but also in orbital adipocytes, fibroblasts, and lymphocytes. Orbital tissues could therefore be targets of an autoimmune reaction in thyroid disease, ${ }^{18}$ although there is a subset of patients with TAO without hyperthyroidism. The assessment of thyroid dysfunction is currently based on serum T3, free T4, and TSH levels (TFT). However, diagnosis of euthyroid TAO using TFT only without a clinical history may include patients whose GD is controlled by antithyroid drug (ATD) treatment, as well as those who had been in remission after cessation of treatment. In addition, ocular symptoms may appear before diagnosis of GD. Because it is unknown whether euthyroid TAO is an independent disease from GD, definitive criteria for euthyroid TAO diagnosis have still not been well established. Kazuo et $a^{3}$ defined euthyroid TAO as TAO with normal thyroid function without any history of hyperthyroidism.

Because TAO has unique ocular symptoms, its diagnosis is relatively easy. However, if there is no knowledge about a history of hyperthyroidism and possible abnormalities in the TFT, there could be increased burdens on both clinicians and patients in terms of time, expense, and delay of proper treatment. Additional tests for other orbital diseases, which could cause isolated proptosis, lid edema and diplopia, might be necessary. Therefore, knowledge of the clinical characteristics of euthyroid TAO could be valuable in diagnosing the disease.

There is a scarcity of reports that systematically investigate the phenotype of euthyroid TAO in Asians. Although Kazuo $\mathrm{et}^{\mathrm{al}} \mathrm{l}^{3}$ reported that exophthalmos (57.1\%) occurred less frequently than lid retraction (74.3\%) or EOM dysfunction (74.3\%) in Japanese euthyroid TAO patients, the authors studied the prevalence of ocular symptoms only with patients diagnosed with this disorder.

In the present study, we compared the clinical aspects of euthyroid TAO with a large subset of hyperthyroid TAO patients. As a result, we found that the ratio of unilateral involvement was higher in euthyroid TAO patients $(79.2 \%)$ than in hyperthyroid TAO patients (27.3\%). Lid retraction was the most commonly observed ocular sign in euthyroid TAO and more commonly observed than in hyperthyroid TAO (91.7\% vs 66.2\%, respectively, $P=0.014)$. Among the 24 patients, lid 
retraction was not observed in only 2 patients. We previously reported patients with papillary micro carcinoma who presented with TAO in an euthyroid state. ${ }^{18}$ Interestingly, unilateral ocular involvement was observed in four of these five TAO patients.

The result that clinical manifestation of euthyroid TAO was less active and severe was similar to the result by Eckstein et al ${ }^{10}$ who studied Caucasian patients. In the present study, the durations of ocular symptoms were not different between the two groups (median duration 3 months, $P=0.733$ ). Because the duration of TAO ocular symptoms, which is the X-axis of the Rundle' curve, ${ }^{19}$ greatly influences clinical activity and/or severity, this data helps to increase the reliability of our study results. Furthermore, we compared clinical aspects of euthyroid TAO in 10 patients in remission, who were a subgroup of hyperthyroid TAO patients. Most notably, there was not a significant difference in CAS and modified NOSPECS scores between the two groups. These results support the hypothesis that clinical manifestation of TAO is influenced by thyroid function. ${ }^{19}$

It was expected that TRAb would be used as a standard criteria in diagnosing euthyroid TAO. TRAb titer, however, was observed to be very low in the euthryoid and hypothyroid patients. TRAb levels could be affected by environmental factors such as peripheral thyroid function. ${ }^{20-22}$ In addition, TRAb was reported to decrease over time after the occurrence of TAO. There are several studies reporting variations of TRAb over time, ${ }^{16,23}$ and one of them showed that TBII levels were markedly decreased over time regardless of a mild or severe course of GO. ${ }^{16}$ Thus, the conversion from positive to negative results might have occurred if TRAb measurements were delayed. Euthyroid TAO with TRAb values, which were negative, has also been reported. ${ }^{24,25}$

In the present study, there were only four people in the group with euthyroid TAO whose ocular symptoms had started more than 12 months previously. Interestingly, both TRAb assays were negative in three patients (75\%). However, of the remaining 20 patients whose ocular symptoms had been less than 12 months, the TBII assay was positive for $42.1 \%(8 / 19)$ and the TSI assay was positive in all the remaining patients (14/14). Therefore, we recommend performing TRAb measurements as early as possible when typical symptoms or signs of TAO are observed. Our results also support a previous report that in Asians, TSI measurement is a more sensitive marker of euthyroid TAO than TBII measurements. ${ }^{10}$

Among the three patients in whom TBII/TSI assays were negative, the patient presenting with diplopia and unilateral proptosis did not show typical symptoms or signs of orbital myositis such as acute pain exacerbated by eye movement. The CT scan revealed right inferior and medical rectus enlargement without anterior tendon involvement. However, the possibility of atypical myositis should be addressed because approximately half of the cases of orbital myositis may not have any tendon involvement. ${ }^{26,27}$

A routine TFT might be recommended in euthyroid TAO. Kazuo et $a l^{3}$ reported 7 patients among 35 with euthyroid TAO whose TRAb was over $5000 \%$. Later, hyperthyroidism occurred in one patient and Hashimoto's disease in two patients. In our study, subclinical hypothyroidism was observed in 3 out of 24 euthyroid TAO patients. The remaining 21 patients did not show any changes in the TFT. Although the euthyroid condition was maintained in most of the patients, the possibility that thyroid function deteriorated still exists.

The present study examined the specific ocular manifestations of euthyroid TAO in Asians, finding a difference between euthyroid and hyperthyroid TAO patients. Furthermore, we discovered that the TSI assay was more sensitive than the TBII assay in diagnosis of euthyroid TAO. Our results would be helpful in early diagnosis and proper management of euthyroid TAO. Further research is still required to elucidate pathogenic mechanisms of euthyroid TAO in systemic autoimmunity that does not provoke hyperthyroidism.

\section{Summary}

What was known before

- Euthyroid TAO is rare and often difficult to diagnose, because of the absence of the standard criteria and confirmatory diagnostic tests.

- Because most cases involving euthyroid TAO showed no thyroid abnormalities except testing positive for thyroidstimulating hormone receptor antibodies (TRAbs), the clinical significance of TRAb as a criterion in the diagnosis of euthyroid TAO has been suggested.

\section{What this study adds}

- The present study examined the clinical manifestations and TRAb levels of euthyroid TAO in Asians, finding a difference between euthyroid and hyperthyroid TAO patients.

- TSI bioassay was more sensitive than the TBII assay in diagnosis of euthyroid TAO, and TRAb levels were significantly lower in euthryoid TAO than in hyperthyroid TAO.

- In patients with euthyroid TAO in whom TSI was measured within 1 year of initial onset of TAO symptoms, the positive rate of TSI bioassay was $100 \%$.

\section{Conflict of interest}

The authors declare no conflict of interest. 


\section{Acknowledgements}

This study was supported by a grant from the College of Medicine, Yonsei University (6-2010-0051).

\section{References}

1 Werner SC. Euthyroid patients with early eye signs of Graves' disease; their responses to L-triiodothyronine and thyrotropin. Am J Med 1955; 18(4): 608-612.

2 Liddle GW, Heyssel RM, McKenzie JM. Graves' disease without hyperthyroidism. Am J Med 1965; 39(5): 845-848.

3 Kazuo K, Fujikado T, Ohmi G, Hosohata J, Tano Y. Value of thyroid stimulating antibody in the diagnosis of thyroid associated ophthalmopathy of euthyroid patients. $\mathrm{Br} J$ Ophthalmol 1997; 81(12): 1080-1083.

4 Eckstein AK, Lax H, Losch C, Glowacka D, Plicht M, Mann $\mathrm{K}$ et al. Patients with severe Graves' ophthalmopathy have a higher risk of relapsing hyperthyroidism and are unlikely to remain in remission. Clin Endocrinol (Oxf) 2007; 67(4): 607-612.

5 Marcocci C, Bartalena L, Bogazzi F, Panicucci M, Pinchera A. Studies on the occurrence of ophthalmopathy in Graves' disease. Acta Endocrinol (Copenh) 1989; 120(4): 473-478.

6 Khoo DH, Eng PH, Ho SC, Tai ES, Morgenthaler NG, Seah LL et al. Graves' ophthalmopathy in the absence of elevated free thyroxine and triiodothyronine levels: prevalence, natural history, and thyrotropin receptor antibody levels. Thyroid 2000; 10(12): 1093-1100.

7 Watanabe M, Iwatani Y, Kashiwai T, Iijima T, Fujikado T, Amino N. Euthyroid Graves' disease showing no thyroid abnormalities except positive thyroid-stimulating antibody (TSAb): two case reports. J Intern Med 1995; 238(4): 379-384.

8 Hornez N, Morell-Dubois S, Woillez JP, Queyrel V, Charlanne H, Launay D et al. Euthyroid Graves' disease: a case report. Rev Med Interne 2009; 30(11): 988-990.

9 Kubo T, Toki J, Kado Y, Kurihara M, Moriwake T, Kanzaki S et al. Thyroid-stimulating antibody in a patient with euthyroid Graves' disease. Endocr J 2000; 47(2): 197-201.

10 Eckstein AK, Losch C, Glowacka D, Schott M, Mann K, Esser J et al. Euthyroid and primarily hypothyroid patients develop milder and significantly more asymmetrical Graves ophthalmopathy. Br J Ophthalmol 2009; 93(8): 1052-1056.

11 Goh SY, Ho SC, Seah LL, Fong KS, Khoo DH. Thyroid autoantibody profiles in ophthalmic dominant and thyroid dominant Graves' disease differ and suggest ophthalmopathy is a multiantigenic disease. Clin Endocrinol (Oxf) 2004; 60(5): 600-607.

12 Kim IT, Choi JB. Normal range of exophthalmos values on orbit computerized tomography in Koreans. Ophthalmologica 2001; 215(3): 156-162.
13 Woo KI, Kim YD, Lee SY. KSOPRS. The clinical characteristics of thyroid orbitopathy in thyroid dysfunction patients in Korea. J Korean Ophthalmol Soc 2001; 49(9): 1387-1396.

14 Sleep TJ, Manners RM. Interinstrument variability in Hertel-type exophthalmometers. Ophthal Plast Reconstr Surg 2002; 18(4): 254-257.

15 Mourits MP, Prummel MF, Wiersinga WM, Koornneef L. Clinical activity score as a guide in the management of patients with Graves' ophthalmopathy. Clin Endocrinol (Oxf) 1997; 47(1): 9-14.

16 Eckstein AK, Plicht M, Lax H, Neuhauser M, Mann K, Lederbogen $\mathrm{S}$ et al. Thyrotropin receptor autoantibodies are independent risk factors for Graves' ophthalmopathy and help to predict severity and outcome of the disease J Clin Endocrinol Metab 2006; 91(9): 3464-3470.

17 Gupta JS, Jain IS, Kumar K. Lid-retraction secondary to contralateral ptosis. Br J Ophthalmol 1964; 48: 626-627.

18 Yoon JS, Lew H, Park JS, Nam KH, Lee SY. Papillary thyroid carcinoma with thyroid-associated orbitopathy in a euthyroid state. Ophthal Plast Reconstr Surg 2007; 23(3): 187-191.

19 Bartley GB. Rundle and his curve. Arch Ophthalmol 2011; 129(3): 356-358.

20 De Bellis A, Bizzarro A, Conte M, Coronella C, Solimeno S, Perrino $S$ et al. Relationship between longitudinal behaviour of some markers of eye autoimmunity and changes in ocular findings in patients with Graves' ophthalmopathy receiving corticosteroid therapy. Clin Endocrinol (Oxf) 2003; 59(3): 388-395.

21 Laurberg P, Wallin G, Tallstedt L, Abraham-Nordling M, Lundell G, Torring O. TSH-receptor autoimmunity in Graves' disease after therapy with anti-thyroid drugs, surgery, or radioiodine: a 5-year prospective randomized study. Eur J Endocrinol 2008; 158(1): 69-75.

22 Takamura Y, Nakano K, Uruno T, Ito Y, Miya A, Kobayashi $\mathrm{K}$ et al. Changes in serum TSH receptor antibody (TRAb) values in patients with Graves' disease after total or subtotal thyroidectomy. Endocr I 2003; 50(5): 595-601.

23 Maugendre D, Massart C. Clinical value of a new TSH binding inihibitory activity assay using human TSH receptors in the follow-up of antithyroid drug treated Graves' disease. Comparison with thyroid stimulating antibody bioassay. Clin Endocrinol (Oxf) 2001; 54(1): 89-96.

24 Cakir M. Euthyroid Graves' ophthalmopathy with negative autoantibodies. J Natl Med Assoc 2005; 97(11): 1547-1549.

25 Paunkovic J, Paunkovic N. Does autoantibody-negative Graves' disease exist? A second evaluation of the clinical diagnosis. Horm Metab Res 2006; 38(1): 53-56.

26 Mannor GE, Rose GE, Moseley IF, Wright JE. Outcome of orbital myositis. Clinical features associated with recurrence. Ophthalmology 1997; 104(3): 409-414.

27 Mombaerts I, Koornneef L. Current status in the treatment of orbital myositis. Ophthalmology 1997; 104(3): 402-408. 